\title{
TROTTER'S PRODUCT FORMULA FOR SEMIGROUPS GENERATED BY QUASILINEAR ELLIPTIC OPERATORS
}

\author{
MICHIAKI WATANABE
}

\begin{abstract}
Trotter's product formula is given for nonlinear semigroups in $L^{1}\left(R^{N}\right)$ generated by quasilinear operators of the form $\Delta \phi$, where $\phi$ is a suitable function: formally $\exp (t \Delta \phi) u=\lim _{h \downarrow 0}\left\{\exp \left(h \Delta \phi_{1}\right) \cdots \exp \left(h \Delta \phi_{k}\right)\right\}^{[t / h]} u$, where $\phi=\phi_{1}+\cdots+\phi_{k}$. The proof is carried out by a new method for construction of a semigroup with generator $\Delta \phi$ in $L^{1}\left(R^{N}\right)$.
\end{abstract}

1. Introduction and main theorem. Let $\phi$ be a differentiable function on $R$ with $\phi(0)=0$ such that $\phi^{\prime}$ is nonnegative and bounded on every bounded subinterval of $R$. Let $\{T(t): t>0\}$ be the strongly continuous semigroup in the Banach space $L^{1}\left(R^{N}\right)$ with norm $\|\cdot\|_{1}$ defined by

$$
T(t) u(x)=(4 \pi t)^{-N / 2} \int_{R^{N}} e^{-|x-y|^{2} /(4 t)} u(y) d y .
$$

Then the infinitesimal generator $A$ of it is the Laplacian $\Delta=\sum_{i=1}^{N} \partial^{2} / \partial x_{i}^{2}$ in $L^{1}\left(R^{N}\right)$. We consider a quasilinear operator $A_{\phi}$ as an operator $\Delta \phi$ in $L^{1}\left(R^{N}\right)$ defined by $A_{\phi} u=A \cdot \phi(u)$ for $u \in D\left(A_{\phi}\right), D\left(A_{\phi}\right)=\left\{u \in L^{1}\left(R^{N}\right) \cap L^{\infty}\left(R^{N}\right): \phi(u) \in\right.$ $D(A)\}$.

We begin our theory with the generation of a nonlinear semigroup $\left\{S_{\phi}(t): t>0\right\}$ in terms of $A_{\phi}$ from the idea of

$$
h^{-1}(u(t+h, x)-u(t, x))=L^{-1}(T(L)=I) \phi(u(t, x)),
$$

which has been employed in $[\mathbf{3}]$, however, as an approximation scheme for the quasilinear parabolic equation $\partial u / \partial t=\Delta \phi(u)$. We construct $\left\{S_{\phi}(t): t>0\right\}$ by means of the operator $C_{h, m}$ defined by

$$
C_{h, m} u=u+h L^{-1}(T(L)-I) \phi(u)
$$

with $h>0$ and $L=h \cdot \sup _{|r| \leq m} \phi^{\prime}(r)$ for a positive integer $m$, and do not appeal to any result concerning the semilinear equation $\phi^{-1}(u)-\Delta u=f($ see $[\mathbf{2}]$ with $[\mathbf{1}]$ ).

The above method enables us not only to give a new proof of the generation but also to deduce some properties of the semigroup $\left\{S_{\phi}(t): t>0\right\}$. As a consequence we obtain Trotter's product formula as follows.

THEOREM. Let $\phi_{j}, j=1, \ldots, k$, be functions on $R$ satisfying the condition for $\phi$ stated in the beginning of the present paper. Let $\left\{S_{\phi_{j}}(t): t>0\right\}, j=1, \ldots, k$, and $\left\{S_{\phi}(t): t>0\right\}$ be the semigroups generated by $A_{\phi_{j}}, j=1, \ldots, k$, and $A_{\phi}$ with $\phi=\phi_{1}+\cdots+\phi_{k}$, respectively.

Received by the editors July 29, 1983 and, in revised form, November 21, 1983.

1980 Mathematics Subject Classification. Primary 47H20, 35K55, 65J15.

Key words and phrases. Nonlinear semigroup, Trotter's product formula, quasilinear operator. 
Then, for every $u \in L^{1}\left(R^{N}\right) \cap L^{\infty}\left(R^{N}\right)$,

$$
\left\{S_{\phi_{1}}(h) \cdots S_{\phi_{k}}(h)\right\}^{[t / h]} u \rightarrow S_{\phi}(t) u \quad \text { in } L^{1}\left(R^{N}\right) \text { as } h \downarrow 0
$$

uniformly on every bounded subinterval of $[0, \infty)$.

The proof of the theorem is obtained by demonstrating that for every $\lambda>0$ and $u \in L^{1}\left(R^{N}\right) \cap L^{\infty}\left(R^{N}\right)$,

$$
\left(I-\lambda h^{-1}\left(S_{\phi_{1}}(h) \cdots S_{\phi_{k}}(h)-I\right)\right)^{-1} \rightarrow\left(I-\lambda A_{\phi}\right)^{-1} u
$$

in $L^{1}\left(R^{N}\right)$ as $h \downarrow 0$, and by then applying Brezis-Pazy's convergence theorem [5, Theorem 3.2]. Such a type of convergence as (1.3) has been discussed for nonlinear semigroups mainly in Hilbert spaces (see e.g. [4 and 8]). Recently Coron [6] established various product formulas in $L^{1}\left(R^{N}\right)$ for semigroups generated by quasilinear differential operators of first order.

2. Construction of $\left\{S_{\phi}(t): t>0\right\}$. We begin this section with a lemma, of which we will make frequent use. Let $X_{m}$, for a positive integer $m$, be the totality of $u \in L^{1}\left(R^{N}\right) \cap L^{\infty}\left(R^{N}\right)$ such that $\|u\|_{\infty} \leq m$, and put $X_{0}=\bigcup_{m=1}^{\infty} X_{m}$. Then, $X_{0}$ equals $L^{1}\left(R^{N}\right) \cap L^{\infty}\left(R^{N}\right)$, a dense subspace of $L^{1}\left(R^{N}\right)$.

We consider a family $\{U(h): h>0\}$ of operators mapping $X_{m}$ into itself for $m \geq 1$, and say that it satisfies the condition $(\mathrm{C})_{m}$ if

(i) $\|U(h) u-U(h) v\|_{1} \leq\|u-v\|_{1}$,

(ii) $\|U(h) u\|_{p} \leq\|u\|_{p}(p=1, \infty)$,

(iii) $U(h) u_{y}=(U(h) u)_{y}$ for $y \in R^{N}$ where $u_{y}(x)=u(x+y)$,

(iv) $\int_{R^{N}} \operatorname{sgn}(u) \cdot h^{-1}(U(h)-I) u f(x) d x \leq C_{m}\|u\|_{1}\|\Delta f\|_{\infty}$ for all $h>0, u, v \in$ $X_{m}$ and a positive constant $C_{m}$, where $f$ is an arbitrary nonnegative bounded function on $R^{N}$ with $\Delta f \in L^{\infty}\left(R^{N}\right)$.

LEMMA 2.1. If a family $\{U(h): h>0\}$ satisfies the condition $(\mathrm{C})_{m}$, then $J_{\lambda, h}=\left(I-\lambda h^{-1}(U(h)-I)\right)^{-1}$ is well defined for every $\lambda>0$, maps $X_{m}$ into itself, and satisfies, for $u, v \in X_{m}$,

(1) $\left\|J_{\lambda, h} u-J_{\lambda, h} v\right\|_{1} \leq\|u-v\|_{1}$,

(2) $\left\|J_{\lambda, h} u\right\|_{p} \leq\|u\|_{p}(p=1, \infty)$,

(3) the set $\left\{J_{\lambda, h} u: h>0\right\}$ is precompact in $L^{1}\left(R^{N}\right)$.

Proof. For a given $u \in X_{m}, J_{\lambda, h} u$ exists as a unique fixed point of the transformation from the closed convex subset $X_{m}$ of $L^{1}\left(R^{N}\right)$ into itself: $v \rightarrow$ $h(\lambda+h)^{-1} u+\lambda(\lambda+h)^{-1} U(h) v$. Clearly (1), (2) and, in particular,

$$
\left\|\left(J_{\lambda, h} u\right)_{y}-J_{\lambda, h} u\right\|_{1} \leq\left\|u_{y}-u\right\|_{1} \quad \text { for } y \in R^{N}
$$

hold. Replacing $u$ in (iv) by $J_{\lambda, h} u$, we have

$$
\int_{R^{N}}\left|J_{\lambda, h} u\right| f(x) d x \leq \int_{R^{N}}|u| f(x) d x+C_{m}\|u\|_{1}\|\Delta f\|_{\infty} .
$$

Putting $f(x)=g(2|x| / \rho-1)(\rho>0)$ in the above, we obtain

$$
\begin{aligned}
& \int_{|x|>\rho}\left|J_{\lambda, h} u\right| d x \\
& \quad \leq \int_{|x|>\rho / 2}|u| d x+\max \left\{4 \rho^{-2}, 2 \rho^{-1}\right\} \lambda C_{m}\|u\|_{1}\left(\left\|g^{\prime \prime}\right\|_{\infty}+(N-1)\left\|g^{\prime}\right\|_{\infty}\right),
\end{aligned}
$$


where $g$ is a function of class $C^{2}: R \rightarrow[0,1]$ with values 0 for $r \leq 0$ and 1 for $r \geq 1$.

Thus, by Fréchet-Kolmogorov theorem, $(2.1),(2.2)$ and $(2)(p=1)$ imply that the set $\left\{J_{\lambda, h} u: h>0\right\}$ is precompact in $L^{1}\left(R^{N}\right)$. Q.E.D.

To construct $\left\{S_{\phi}(t): t>0\right\}$, we will deal with the operator $C_{h, m}$ defined on $X_{m}$ by (1.2) for each fixed $m \geq 1$.

LEMMA 2.2. For each $m \geq 1$, the family $\left\{C_{h, m}: h>0\right\}$ satisfies the condition $(\mathrm{C})_{m}$ with $C_{m}=\sup _{|r| \leq m} \phi^{\prime}(r)$.

PROOF. Since $r-h L^{-1} \phi(r)$ is nondecreasing in $r$ and hence

$$
\left|r-s-h L^{-1}(\phi(r)-\phi(s))\right|+h L^{-1}|\phi(r)-\phi(s)|=|r-s| \quad \text { for } r, s \in[-m, m],
$$

$C_{h, m}$ satisfies (i) and (ii). The validity of (iii) is clear from (1.1) and (1.2). It remains to show (iv). Since $h^{-1}\left(C_{h, m}-I\right)=L^{-1}(T(L)-I) \phi(\cdot)$,

$$
\operatorname{sgn}(u) \cdot h^{-1}\left(C_{h, m}-I\right) u \leq L^{-1}(T(L)-I)|\phi(u)|
$$

holds. Multiplication by $f(x)$ and integration of this inequality over $R^{N}$ gives

$$
\int_{R^{N}} \operatorname{sgn}(u) \cdot h^{-1}\left(C_{h, m}-I\right) u f(x) d x \leq \int_{R^{N}}|\phi(u)| L^{-1}(T(L)-I) f(x) d x \text {. Q.E.D. }
$$

Proposition 2.3. $A_{\phi}$ is a dissipative operator with domain $D\left(A_{\phi}\right)$ dense in $L^{1}\left(R^{N}\right)$ satisfying the range condition

$$
R\left(I-\lambda A_{\phi}\right) \supset X_{0} \text { for any } \lambda>0 .
$$

Moreover, for any $m \geq 1,\left(I-\lambda A_{\phi}\right)^{-1}$ maps $X_{m}$ into itself and satisfies, for evęry $u, v \in X_{m}$,

$$
\begin{gathered}
\left\|\left(I-\lambda A_{\phi}\right)^{-1} u-\left(I-\lambda A_{\phi}\right)^{-1} v\right\|_{1} \leq\|u-v\|_{1}, \\
\left\|\left(I-\lambda A_{\phi}\right)^{-1} u\right\|_{p} \leq\|u\|_{p} \quad(p=1, \infty),
\end{gathered}
$$

and

$$
\left(I-\lambda h^{-1}\left(C_{h, m}-I\right)\right)^{-1} u \rightarrow\left(I-\lambda A_{\phi}\right)^{-1} u \quad \text { in } L^{1}\left(R^{N}\right)
$$

as $h \downarrow 0$.

PROOF. Let $u \in X_{m}$ for an arbitrary $m \geq 1$ and let $\left\{h_{n}\right\}_{n=1}^{\infty}$ be a sequence such that $h_{n} \downarrow 0$. Then, by Lemma 2.1, Lemma 2.2 implies that the sequence $\left\{J_{h_{n}, m}^{\lambda} u\right\}_{n=1}^{\infty}$, where $J_{h, m}^{\lambda}=\left(I-\lambda h^{-1}\left(C_{h, m}-I\right)\right)^{-1}$ for $h=h_{n}$, contains a subsequence convergent to some $u_{\lambda, m}$ in $L^{1}\left(R^{N}\right)$. The equality

$$
\begin{aligned}
& \left(I-\mu L^{-1}(T(L)-I)\right)^{-1} \lambda^{-1}\left(J_{h, m}^{\lambda} u-u\right) \\
& \quad=\mu^{-1}\left\{\left(I-\mu L^{-1}(T(L)-I)\right)^{-1}-I\right\} \phi\left(J_{h, m}^{\lambda} u\right) \text { for } \mu>0
\end{aligned}
$$

and the fact that, for every $v \in L^{1}\left(R^{N}\right)$,

$$
\left(I-\mu t^{-1}(T(t)-I)\right)^{-1} v \rightarrow(I-\mu A)^{-1} v \quad \text { in } L^{1}\left(R^{N}\right)
$$

as $t \downarrow 0$ imply that

$$
(I-\mu A)^{-1} \lambda^{-1}\left(u_{\lambda, m}-u\right)=\mu^{-1}\left((I-\mu A)^{-1}-I\right) \phi\left(u_{\lambda, m}\right),
$$


that is, $\left(I-\lambda A_{\phi}\right) u_{\lambda, m}=u$ with $u_{\lambda, m} \in D\left(A_{\phi}\right)$ since $\left\|u_{\lambda, m}\right\|_{\infty} \leq\|u\|_{\infty}$. Thus, the dissipativeness of $A_{\phi}$, which is obtained by letting $t \downarrow 0$ in the inequality

$$
\int_{R^{N}} \operatorname{sgn}\left(v_{1}-v_{2}\right) \cdot t^{-1}(T(t)-I)\left(\phi\left(v_{1}\right)-\phi\left(v_{2}\right)\right) d x \leq 0
$$

for $v_{1}, v_{2} \in D\left(A_{\phi}\right)$, implies that $u_{\lambda, m}=\left(I-\lambda A_{\phi}\right)^{-1} u$.

Finally, we prove that $D\left(A_{\phi}\right)$ is dense in $L^{1}\left(R^{N}\right)$. To this end it suffices to show that for any $u \in X_{m}, m \geq 1,\left(I-\lambda A_{\phi}\right)^{-1} u$ converges in $L^{1}\left(R^{N}\right)$ to $u$ as $\lambda \downarrow 0$. Since $(2.1),(2.2)$ and $(2)(p=1)$ remain true with $J_{\lambda, h} u$ and $C_{m}$ replaced by $\left(I-\lambda A_{\phi}\right)^{-1} u$ and $\sup _{|r| \leq m} \phi^{\prime}(r)$, respectively, we can prove by a similar method to that used in the proof of Lemma 2.1 that the set $\left\{\left(I-\lambda A_{\phi}\right)^{-1} u: \lambda>0\right\}$ is precompact in $L^{1}\left(R^{N}\right)$. Therefore for any sequence $\left\{\lambda_{n}\right\}_{n=1}^{\infty}$ such that $\lambda_{n} \downarrow 0$, the sequence $\left\{\left(I-\lambda_{n} A_{\phi}\right)^{-1} u\right\}_{n=1}^{\infty}$ contains a subsequence convergent to some $w$ in $L^{1}\left(R^{N}\right)$. The equality $(2.5)$ with $u_{\lambda, m}$ replaced with $\left(I-\lambda A_{\phi}\right)^{-1} u$ implies that $(I-\mu A)^{-1}(w-u)=0$ for $\mu>0$. Q.E.D.

3. Properties of $\left\{S_{\phi}(t): t>0\right\}$. In the preceding section we have proved that $A_{\phi}$ generates a semigroup $\left\{S_{\phi}(t): t>0\right\}$ in the sense of Crandall-Liggett [7, Theorem I]. The purpose of this section is to study further properties of $\left\{S_{\phi}(t): t>\right.$ $0\}$ and to give the proof of Theorem. With the aid of the Brezis-Pazy's convergence theorem, the following can be obtained from Proposition 2.3.

Proposition 3.1. For every $t>0, S_{\phi}(t)$ maps $X_{m}$ into itself for any $m \geq 1$ and satisfies, for every $u, v \in X_{m}$,

$$
\left\|S_{\phi}(t) u-S_{\phi}(t) v\right\|_{1} \leq\|u-v\|_{1}, \quad\left\|S_{\phi}(t) u\right\|_{p} \leq\|u\|_{p} \quad(p=1, \infty)
$$

and

$$
C_{h, m}^{[t / h]} u \rightarrow S_{\phi}(t) u \quad \text { in } L^{1}\left(R^{N}\right)
$$

as $h \downarrow 0$ uniformly on every bounded subinterval of $[0, \infty)$.

LEMMA 3.2. For every $m \geq 1,\left\{S_{\phi}(t): t>0\right\}$ satisfies the condition $(\mathrm{C})_{m}$ with $C_{m}=\sup _{|r| \leq m} \phi^{\prime}(r)$.

PROOF. In view of $(3.1)$ we see that $S_{\phi}(t) u_{y}=\left(S_{\phi}(t) u\right)_{y}$ for $y \in R^{N}$ since $C_{h, m} u_{y}=\left(C_{h, m} u\right)_{y}$. It remains to show that, for $u \in X_{m}$,

$$
\int_{R^{N}} \operatorname{sgn}(u) \cdot t^{-1}\left(S_{\phi}(t)-I\right) u f(x) d x \leq \sup _{|r| \leq m} \phi^{\prime}(r) \cdot\|u\|_{1}\|\Delta f\|_{\infty} .
$$

Since $C_{h, m}^{n}-I=\sum_{k=0}^{n-1}\left(C_{h, m}-I\right) C_{h, m}^{k}$ for any positive integer $n$, we have, by Lemma 2.2 ,

$$
\int_{R^{N}} \operatorname{sgn}(u) \cdot\left(C_{h, m}^{n}-I\right) u f(x) d x \leq n h \sup _{|r| \leq m} \phi^{\prime}(r) \cdot\|u\|_{1}\|\Delta f\|_{\infty} .
$$

Putting $n=[t / h]$ and letting $h \downarrow 0$ yields (3.2). Q.E.D.

LEMMA 3.3. For every $u \in X_{0}, \int_{0}^{t} \phi\left(S_{\phi}(r) u\right) d r$ belongs to $D(A)$, and for all $t \geq 0$,

$$
S_{\phi}(t) u-u=A \int_{0}^{t} \phi\left(S_{\phi}(r) u\right) d r \quad \text { in } L^{1}\left(R^{N}\right)
$$


PROOF. Let $u \in X_{m}$ for an arbitrary $m \geq 1$ and let $u_{h}$ be, for $h>0$, the solution in $C\left([0, \infty) ; X_{m}\right)$ of

$$
u(t)=e^{-t / h} u+h^{-1} \int_{0}^{t} e^{-(t-r) / h} C_{h, m} u(r) d r, \quad t \geq 0 .
$$

It is easy to verify that $u_{h}(t)$ is differentiable in $t$ in the topology of $L^{1}\left(R^{N}\right)$ and satisfies

$$
u_{h}(t)-u=L^{-1}(T(L)-I) \int_{0}^{t} \phi\left(u_{h}(r)\right) d r, \quad t \geq 0 .
$$

By a well-known convergence theorem (see e.g. [5, Theorem 3.1]), (2.3) implies that

$$
u_{h}(t) \rightarrow S_{\phi}(t) u \quad \text { in } L^{1}\left(R^{N}\right)
$$

as $h \downarrow 0$ uniformly on every bounded subinterval of $[0, \infty)$. From (3.4) it follows that for any $\mu>0$,

$$
\begin{aligned}
& \left(I-\mu L^{-1}(T(L)-I)\right)^{-1}\left(u_{h}(t)-u\right) \\
& \quad=\mu^{-1}\left\{\left(I-\mu L^{-1}(T(L)-I)\right)^{-1}-I\right\} \int_{0}^{t} \phi\left(u_{h}(r)\right) d r,
\end{aligned}
$$

which together with (2.4) and (3.5) implies (3.3). Q.E.D.

LEMMA 3.4. Under the assumptions of Theorem, the family $\left\{S_{\phi_{1}}(t) \cdots S_{\phi_{k}}(t)\right.$ : $t>0\}$ satisfies the condition $(\mathrm{C})_{m}$ with $C_{m}=\sum_{j=1}^{k} \sup _{|r| \leq m} \phi_{j}^{\prime}(r)$ for every $m \geq 1$.

PROOF. The validity of (i)-(iii) of $(\mathrm{C})_{m}$ is clear from Lemma 3.2. Since

$$
S_{\phi_{1}}(t) \cdots S_{\phi_{k}}(t)-I=\sum_{j=1}^{k}\left(S_{\phi_{j}}(t)-I\right) S_{\phi_{j+1}}(t) \cdots S_{\phi_{k}}(t)
$$

$k$ applications of (3.2) yields

$$
\begin{array}{rl}
\int_{R^{N}} \operatorname{sgn}(u) & \cdot\left(S_{\phi_{1}}(t) \cdots S_{\phi_{k}}(t)-I\right) u f(x) d x \\
\leq t & t \sum_{j=1}^{k} \sup _{|r| \leq m} \phi_{j}^{\prime}(r) \cdot\|u\|_{1}\|\Delta f\|_{\infty} \cdot \text { Q.E.D. }
\end{array}
$$

PROOF OF THEOREM. As was mentioned in the first section, we have only to show (1.3). Let $u \in X_{0}$. Then, $u \in X_{m}$ for some $m \geq 1$. By Lemma 3.4, for any sequence $\left\{h_{n}\right\}_{n=1}^{\infty}$ such that $h_{n} \downarrow 0$, the sequence $\left\{J_{h_{n}}^{\lambda} u\right\}_{n=1}^{\infty}$, where

$$
J_{h}^{\lambda}=\left(I-\lambda h^{-1}\left(S_{\phi_{1}}(h) \cdots S_{\phi_{k}}(h)-I\right)\right)^{-1}
$$

for $h=h_{n}$, contains a subsequence convergent to some $u_{\lambda}$ in $L^{1}\left(R^{N}\right)$. By Lemma 3.3 it holds that, for $h>0$,

$$
\begin{aligned}
\lambda^{-1}\left(J_{h}^{\lambda} u-u\right) & =h^{-1}\left(S_{\phi_{1}}(h) \cdots S_{\phi_{k}}(h)-I\right) J_{h}^{\lambda} u \\
& =h^{-1} \sum_{j=1}^{k}\left(S_{\phi_{j}}(h)-I\right) S_{\phi_{j+1}}(h) \cdots S_{\phi_{k}}(h) J_{h}^{\lambda} u \\
& =A \cdot \sum_{j=1}^{k} h^{-1} \int_{0}^{h} \phi_{j}\left(S_{\phi_{j}}(r) S_{\phi_{j+1}}(h) \cdots S_{\phi_{k}}(h) J_{h}^{\lambda} u\right) d r
\end{aligned}
$$


Since $A$ is closed and $\left\|u_{\lambda}\right\|_{\infty} \leq\|u\|_{\infty}$, (3.6) implies that

$$
\lambda^{-1}\left(u_{\lambda}-u\right)=A \cdot \sum_{j=1}^{k} \phi_{j}\left(u_{\lambda}\right)=A_{\phi} u_{\lambda},
$$

and hence $u_{\lambda}=\left(I-\lambda A_{\phi}\right)^{-1} u$. Q.E.D.

Quite similarly, with the aid of Lemma 2.1 we can also obtain the following formula under the assumptions of Theorem:

$$
\left\{k^{-1}\left(S_{\phi_{1}}(k h)+\cdots+S_{\phi_{k}}(k h)\right)\right\}^{[t / h]} u \rightarrow S_{\phi}(t) u \text { in } L^{1}\left(R^{N}\right)
$$

as $h \downarrow 0$ for $u \in L^{1}\left(R^{N}\right) \cap L^{\infty}\left(R^{N}\right)$, uniformly on every bounded subinterval of $[0, \infty)$. In fact, the family $\left\{k^{-1}\left(S_{\phi_{1}}(k t)+\cdots+S_{\phi_{k}}(k t)\right): t>0\right\}$ satisfies the condition $(\mathrm{C})_{m}$ with $C_{m}=\sum_{j=1}^{k} \sup _{|r| \leq m} \phi_{j}^{\prime}(r)$.

\section{REFERENCES}

1. Ph. Bénilan, H. Brézis and M. G. Crandall, A semilinear elliptic equation in $L^{1}\left(R^{N}\right)$, Ann. Scuola Norm. Sup. Pisa Cl. Sci. (4) 2 (1975), 523-555.

2. Ph. Bénilan and M. G. Crandall, The continuous dependence on $\phi$ of solutions of $u_{t}-\Delta \phi(u)=0$, Indiana Univ. Math. J. 30 (1981), 161-177.

3. A. E. Berger, H. Brézis and C. W. Rogers, A numerical method for solving the problem $u_{t}-$ $\Delta f(u)=0$, RAIRO Anal. Numér. 13 (1979), 297-312.

4. H. Brézis and A. Pazy, Semigroups of nonlinear contractions on convex sets, J. Funct. Anal. 6 (1970), 237-281.

5. __ Convergence and approximation of semigroups of nonlinear operators in Banach spaces, J. Funct. Anal. 9 (1972), 63-74.

6. J-M. Coron, Formules de Trotter pour une équation d'évolution quasilinéaire du 1 er ordre, J. Math. Pures Appl. 9 (1982), 91-112.

7. M. G. Crandall and T. M. Liggett, Generation of semigroups of nonlinear transformation on general Banach spaces, Amer. J. Math. 93 (1971), 265-298.

8. T. Kato and K. Masuda, Trotter's product formula for nonlinear semi-groups generated by the subdifferentials of convex functionals, J. Math. Soc. Japan 30 (1978), 169-178.

9. S. Oharu and T. Takahashi, A convergence theorem of nomlinear semigroups and its application to first order quasilinear equations, J. Math. Soc. Japan 26 (1974), 124-160.

FACUlty of General EduCation, Nitgata University, IKarashi 2-8050, NiIgAta, JAPAN 950-21 